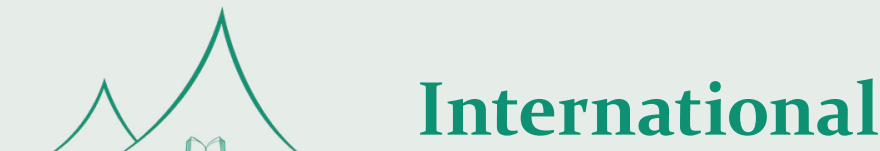 madridge Journal of Physics: Study and Research interconnecting Scientific World
}

Research Article

Open Access

\section{The Mechanism of the Gravitational Force and the Balance of the Universe}

\author{
Emad Y Moawad ${ }^{*}$
}

Researcher graduated, Department of Engineering, Ain Shams University, Cairo, Egypt

\section{Article Info}

\section{*Corresponding author:}

Emad Y Moawad

Researcher graduated

Department of Engineering

Ain Shams University

Alhegaz Street, Alnozha

Egypt

E-mail: emadmoawad@hotmail.com

Received: May 11, 2018
Accepted: May 18, 2018
Published: May 23, 2018

Citation: Moawad EY. The Mechanism of the Gravitational Force and the Balance of the Universe. Int J Phys Stud Res. 2018; 1(1): 1-5. doi: 10.18689/ijpsr-1000101

\section{Copyright: () 2018 The Author(s). This work is licensed under a Creative Commons Attribution 4.0 International License, which permits unrestricted use, distribution, and reproduction in any medium, provided the original work is properly cited.}

Published by Madridge Publishers

\begin{abstract}
Theory of the relative image provides a perception about the mechanism by which the gravitational forces act on the cosmological bodies achieving the equilibrium of our universe.

The cosmological objects are subjected to variable spring forces of simple harmonic motion (compression and stretching) resulting in their motion along wavy paths. These spring forces act on the cosmological objects through gravitational waves that spread perpendicular to the wavy paths of those objects. The total work (W) done by these variable spring forces on the cosmological objects when it moves from $(-A \rightarrow+A)$ is zero, where $A$ is the amplitude of the simple harmonic motion with no energy transfer or loss leaving that isolated system. Along the wavy path the velocity of the cosmological object varies inversely with the acting variable spring force. For each gravitational wave produced by the cosmological body along the wavy path by certain velocity during compression there is identical one (same amplitude and frequency) produced by the same velocity in opposite direction during stretching. Accordingly, the gravitational waves produced during compression destruct those produced during stretching preserving self-sustaining of frictionless system.
\end{abstract}

Keywords: Gravitational force; Centripetal forces; Equilibrium; Relative velocity; Cosmological bodies

\section{Introduction}

Theory of the relative image [1] provides a perception about the mechanism by which the gravitational forces act on the cosmological bodies achieving the equilibrium of our universe. Each body moves around a second one along a path has different detected shape according to the relative velocity between the observer and the second body. In the absence of this relative velocity or when the observer detects the first body orbiting the second body from the second body itself (1st image), this path appears elliptical path and the second body appears stationary lies on its center. The force acting on the first body in the 1st image appears constant centripetal force and always attract the first body towards the second one at the center of the elliptical path and equivalent to equation, where $(m),(v)$ are the mass and velocity of the first body and $(r)$ is the radius of the elliptical path as already known by the latter and confirmed by the former. In the presence of relative velocity between the observer and the second body (2nd image), the path of the first body appears wavy while the second body appears moving along another elliptical path around a third body. The elliptical path appears to the second observer in the 2nd image is the axis of the wavy path of the first body. The amplitude (A) and periodic time (T) of the wavy path in the 2 nd image are equals to the radius ( $r$ ) and $(\mathrm{T})$ of the elliptical path detected by the first observer in the 1st image. Accordingly, 
the first body appears in the 2nd image has two motions; the first along a wavy path alone and the second as a companion for the second body along an elliptical path orbiting the third one. Figure 6a. and Figure 6b. show the 1st and 2nd images respectively for the first body (silver) orbits a second one (blue) in the absence and presence of relative velocity between the observer and the second body respectively.

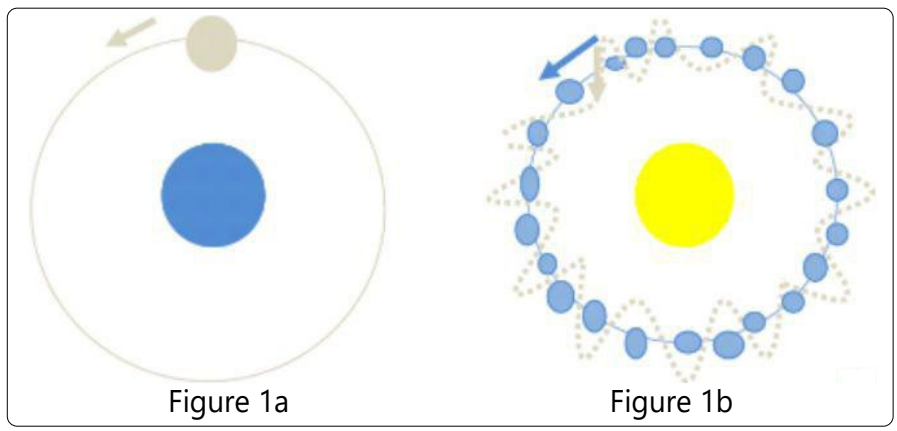

Figure 1a. (1st image) (Observer on the second body (blue)) Figure 1b. (2nd image) (Observer on the third body (yellow) around which the second body (blue) orbits)

\section{Forces acting on the cosmological objects}

These two motions of the first body in the 2nd image is under the action of two forces; the first is variable elastic restoring spring force under which the first body moves in simple harmonic motion along the wavy path towards and away from the elliptical path around which the second body orbits the third one. The magnitude and direction of this spring force is given by $F=-k x$, where $(k)$ is the elastic restoring constant and $(x)$ is the distance between the first body and the elliptical path around which the second body orbits the third one. The negative sign indicates that $(F)$ is in the opposite direction to that of the increase of $(x)$. The maximum value of this force (Fmax $=-m \omega 2 A$ ) is at $x=A$. The second force under which the first body appears moving as a companion for the second body along the elliptical path in the 2 nd image is a constant centripetal force appears always attract both bodies as a one unit towards the third body at the center of the elliptical path similar to that appeared acting on the first body in the 1st image. Similarly, the appeared motion for the first and second bodies in the 2 nd image along the elliptical path orbiting the third body is detected from the third body in the absence of relative velocity between the second observer and the third body. Accordingly, in the presence of relative velocity between a third observer and the third body the elliptical path that seemed to the second observer in the 2nd image is a wavy path in a 3rd image along which the second body moves under variable spring forces. While the wavy path appeared in the 2nd image along which the first body moves is a wavy path of longer wavelength with the same spring forces in the 2nd image. Accordingly, in the orbiting systems the gravitational force acting on the body orbits a Second one is a variable force of reversible direction similar to the spring forces of the simple harmonic motion. Under which the orbiting body moves along a wavy path observed only in the presence of relative velocity between the observer and the second body. Such mechanism of the gravitational forces acting on the cosmological bodies is the perception of theory of the relative image through which the equilibrium of our universe has been achieved.

For instance, in the Earth-Sun system; the moon orbits around the earth under the gravitational force acting on the moon by the earth. The path of this motion is detected elliptically from the earth under a constant centripetal force as shown in Figure 2.

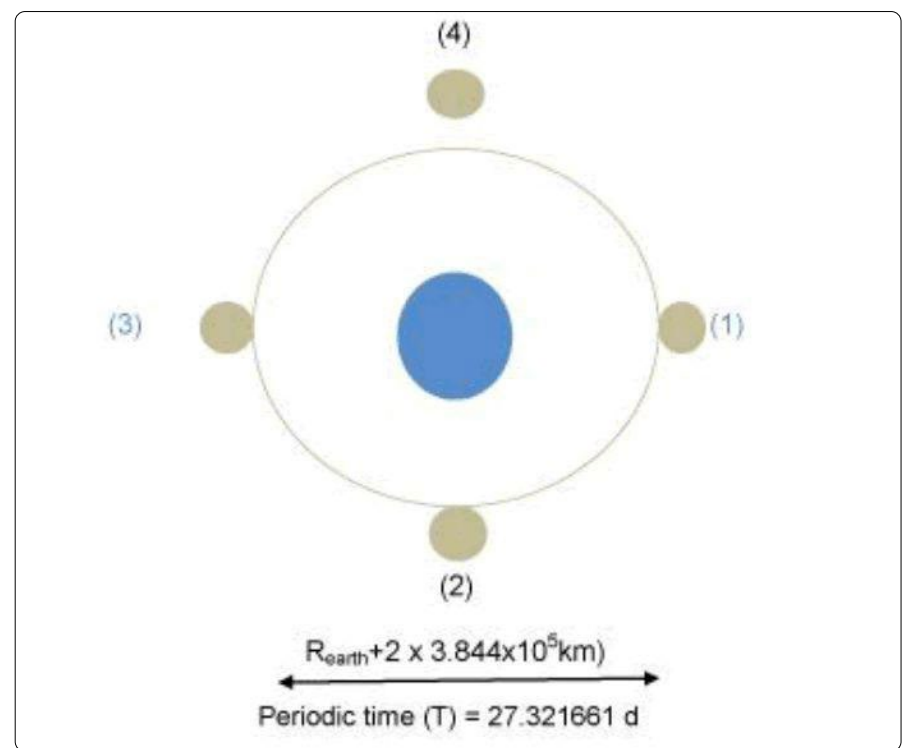

Figure 2. The apparent elliptical image of the path of the Moon with respect to the Earth as observed from the Earth.

The same path is detected from a place of relative velocity with the earth like the sun wavy, under a variable spring force Figure 3.

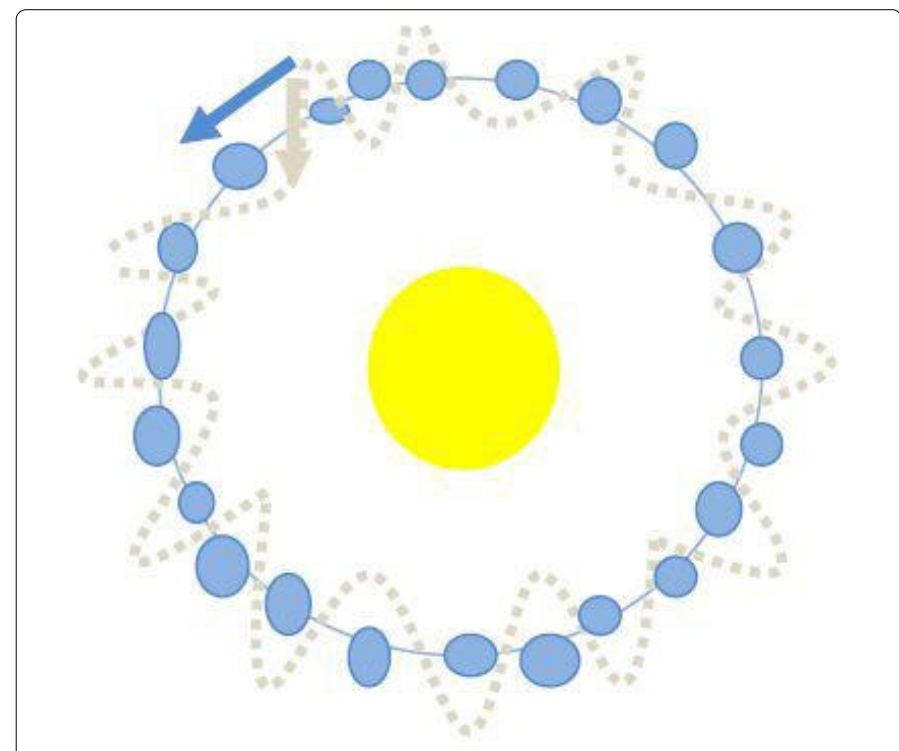

Figure 3. Shows the motion of the moon (silver) along a wavy path (silver) alone and along elliptical path (blue) as a companion for the earth (blue) around the sun (yellow) as observed from the sun.

Since the two images are for a motion of one body during the same period then the energy of the body should be the same in both images. The moon of mass $(m=7.324 \times 10 * 22$ $\mathrm{kg})$ and orbiting with velocity $(\mathrm{v}=1023 \mathrm{~km} / \mathrm{s})$ in the image of the elliptical path around the earth (Figure. 7) of radius $(r=3.844 \times 10 * 8 \mathrm{~m})$, with kinetic energy equation of $3.83 \times 10 * 28$ $\mathrm{j}$ [2]. On the other hand, the total energy (E) of the moon that 
oscillates in the image of the wavy path around the elliptical path of the earth around the sun (Figure. 3) with displacement $(x$; of maximum value of $A$ ) from the equilibrium position which is the elliptical path of the earth around the sun forms a wave of amplitude $(A=3.844 \times 10 * 8 \mathrm{~m})$ with angular velocity $\boldsymbol{\omega}=2 \pi / t=2.6616995310-6 \mathrm{~Hz}$ ) is $1 / 2 \mathrm{KA} 2$ where $\mathrm{k}=\boldsymbol{\omega} 2 \mathrm{~m}$ is the constant of the restoring elastic force exerted on the oscillator) $3.837 \times 10 * 28 \mathrm{j}$. Thus, the energy of the moon along the image of the wavy path around the elliptical path of the earth around the sun observed by the astronomer on the spaceship orbiting nears the sun is $100 \%$ identical to that in the image of the elliptical path around the earth observed by the observer on the earth. On the other hand, the exerted constant force acting on the moon in the image of the elliptical path around the earth is equation, whereas the variable force acting on the moon in the image of the wavy path around the elliptical path of the earth around the sun ( $F=-k x$; with maximum value at $\mathrm{x}=\mathrm{A}$ ) is equation which is also $100 \%$ identical to that of the moon in the image of the elliptical path around the earth.

The restoring elastic (spring) force acting on the moon along its wavy path around the earth is given by- $K x=-k A$ sin wt of maximum value of-KA, where $A$ is the amplitude of the simple harmonic motion equals to the fixed distance between the earth and the moon $(3.844 \times 10 * 8 \mathrm{~m})$. This force varies according to the distance $(x)$ between the moon and the elliptical path of the earth around the sun. The negative sign indicates that the direction of the spring force is opposite to the increase direction of the displacement $(x)$ of the moon from the equilibrium position which is the elliptical path of the earth around the sun.

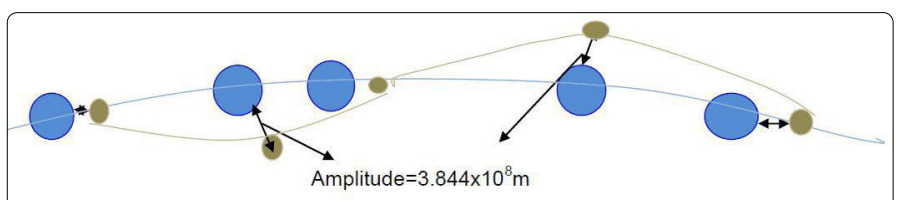

Wave length $(\lambda)=7.031 \times 10^{10} \mathrm{~m}$ and Periodic time $(T)=27.321661 \mathrm{~d}$

$\begin{array}{lllll}\longleftrightarrow \text { (1) (2) } & \text { (3) } & \text { (4) }\end{array}$

Figure 4. shows the wavy image of the path of the Moon with respect to the Earth as traced from the Sun.

From Figure. 4 on the positions of intersection of the wavy and the elliptical paths, the moon acquire its extreme velocity $(\boldsymbol{\omega} \mathrm{A}=1023 \mathrm{~m} / \mathrm{s})$ and is driven by maximum deceleration (- $\boldsymbol{\omega} 2 \mathrm{~A})$ whereas the spring force acting on the moon vanishes as the moon is on the equilibrium position of the spring force $(x=0)$ which is the path of earth around the sun. At those points of intersection between the two paths $(x=0)$ (when the spring forces vanish), the centripetal forces acting on the moon (equation) and the earth equation by the sun are $4 \times 1020 \mathrm{~N}$, $3.54 \times 10 * 22 \mathrm{~N}$ respectively of summation of $3.58 \times 10 * 22 \mathrm{~N}$. While at positions of troughs and crests, $(x=A,-A)$ shown in Figure. 4 (when $\mathrm{t}=\mathrm{T} / 4$ and $3 \mathrm{~T} / 4$ ) the moon velocity instantly vanished, the restoring elastic (spring) force acting on the moon becomes maximum $(K A=2 \times 10 * 20 \mathrm{~N})$ that accelerates the moon once again in direction of the wavy path. Thus, along the whole wavy path the variable spring force acting on the moon is (-mw2Asin $\boldsymbol{\omega t})$ and $\in\left[0 \rightarrow 2 \times 10^{*} 20 \mathrm{~N}\right]$ ) causes the moon to oscillate in the wavy path below and above the elliptical path of the earth around the sun. When the spring force acting on the moon is opposite to the direction of the centripetal forces acting on the moon and the earth by the sun, the moon goes down the elliptical path along the wavy path until the trough $(x=A)$ at which the resultant of forces acting on the moon and the earth decreases to $3.56 \times 10 * 22 \mathrm{~N}$ only. Afterwards, the spring force acting on the moon decreases again $(x<A)$, while the resultant force acting on the moon increases gradually to $3.58 \times 10 * 22 \mathrm{~N}$ at $(x=0)$ once again. When the spring force acting on the moon is in the direction of the centripetal forces acting on the moon and the earth by the sun, the moon goes up the elliptical path along the wavy path until the crest $(x=-A)$ at which the resultant of forces acting on the moon and the earth increases to $3.60 \times 10 * 22 \mathrm{~N}$. Afterwards, the spring force acting on the moon decreases again $(x>-A)$, while the resultant force acting on the moon decreases gradually to $3.58 \times 10 * 22 \mathrm{~N}$ at $(\mathrm{x}=0)$ and so on. Accordingly, the resultant force acting on the moon and the earth by the sun along the whole elliptical path around the sun is not of constant value as postulated by the Newtonian gravitational theory but varies as proved by theory of the relative image with average $(3.58 \times 10 * 22 \mathrm{~N})$ equivalent to that predicted by the Newtonian gravitational theory. Similarly, the earth orbits around the sun under the gravitational force acting on the earth by the sun. The path of this motion is detected elliptically from the sun under a constant centripetal force. The same path is detected from a place of relative velocity with the sun like the Milky-Way wavy under a variable spring force. Figure 5 . shows the moon (silver) and the earth (blue) move along the elliptical path (brown) around the Milky-Way (not shown) as companions for the sun (yellow), and along wavy paths (silver and blue respectively) around the sun as observed from the Milky Way.

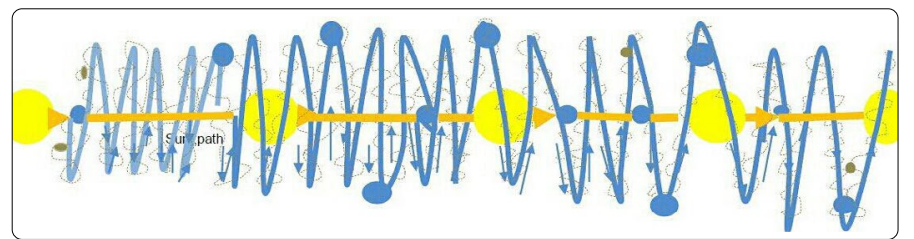

Figure 5. shows the moon (silver) and the earth (blue) move along the elliptical path (brown) around the Milky- Way (not shown) as companions for the sun (yellow), and along wavy paths (silver and blue respectively) around the sun as observed from the Milky Way.

In Figure 5. each of the moon and the sun moves under the action of variable spring forces causes the moon and the earth to oscillate in wavy paths around the elliptical path of the sun around the Milky-Way. Each of these spring forces can be determined as previously shown for the motion of the moon around the earth. Thus, theory of the relative image gives new insights into the working of the Universe. It refutes what has been postulated by either of the Newtonian and general relativity that the gravitational forces acting on the cosmological bodies are constant and centripetal always attractive $[3,4]$. Conversely, it proves that the gravitational forces that act on the cosmological bodies are variable elastic restoring spring forces under which the cosmological bodies move along wavy paths preserving the fixed distances between them which are the amplitudes of their simple harmonic motions. 
However, equilibrium of the cosmological body in any of its relative images has been clarified inseparable from the equilibrium of the whole universe as one unit. All the contents of this unit are bounded by an integrated system of variable elastic restoring (spring) forces of simple harmonic motions (seem constant only due to the observer position). These contents (the cosmological objects) are driven by these variable spring forces along wavy paths, with velocity decrease gradually until they come to the instantaneous rest at the maximum amplitude of the motion then the spring forces reversed in opposite direction with maximum value and change the direction of motion with maximum velocity once again, like oxygen when revives the heart after exhaling to keep life. Statics generates motion that decreases gradually and faints again until revives and so on. Accordingly, velocities of all the contents of our universe are not constant but accelerated and then decelerated in a frictionless isolated system of permanent self-sustaining. Such acceleration and deceleration expresses the balance between the even distribution of the matter along the space-time. Despite of the relative path length that observed differs from image to another for the same motion; the spaces between objects (Amplitudes of the wavy paths) are fixed in all these relative images reflecting a precise system. Thus, theory of the relative image proves that matter and spacetime mutually interact preserving their even distribution and consequently the static of the universe as a one unit. This fact refutes the expanding model as a proposed model for the universe. Conversely, theory of the relative image proves that our universe is like a self-sustaining being always static, while all its inner organs never stop through efficient cycle of acceleration and deceleration without energy loss (path-decay) so that no absolute rest along the spacetime. Such cycles of acceleration and deceleration while the cosmological objects are under compression and then stretching affect the wavelengths of the transmitted gravitational waves across the universe in the direction of motion of these bodies which make it hard to be detected. Furthermore, every gravitational wave has its reversible for self-destruction as shown before (Figure 6) so that no influences occur on the fixed space between objects (Amplitudes of the wavy paths) sustaining the static of the universe. In addition, our suggested self sustaining static universe of none absolute rest along its spacetime explains as previously the observations of the intergalactic redshift, emitted radiations by the black holes and the cosmic microwave background radiation that misled the latter to propose the expansion model for our universe after the Big-Bang.

\section{Produced gravitational waves}

According to theory of the general relativity, the cosmological objects generate gravitational waves during motion that propagate and travelling outward from their source at the speed of light. These gravitational waves transport energy as gravitational radiation, a form of radiant energy similar to electromagnetic radiation [5]. Theory of the relative image has a different point of view regarding the gravitational waves consistent with their tiny detected observations as follows: The cosmological objects are subjected to variable spring forces of simple harmonic motion (compression and stretching) resulting in their motion along wavy paths. These spring forces act on the cosmological objects through gravitational waves that spread perpendicular to the wavy paths of those objects. Accordingly, these gravitational waves express simple harmonic motion of the cosmological objects along their wavy paths under the action of the spring forces. The total energy (E) of the oscillator along its path is given by $\mathrm{E}=$ Potential Energy+Kinetic energy= equation[6]. While, the total work (W) done by these variable spring forces on the cosmological objects when it moves from $(-A \rightarrow+A)$ is zero, where $A$ is the amplitude of the simple harmonic motion with no energy transfer or loss leaving that isolated system [6]. The instantaneous power (P) of the oscillator along these paths is defined by the rate of energy transfer equation where kinetic energy is the energy transfer method in the simple harmonic motion [6]. Thus, no gravitational waves produced when the spring force vanishes at the equilibrium position of the spring when the cosmological body (oscillator) is on the axis of the wave. Along the wavy path the velocity of the oscillator (cosmological object) varies inversely with the acting variable spring force. The oscillator passes the axis of the wave with maximum velocity when the spring force vanishes and comes to instantaneous rest at the crests and the troughs at the maximum value of the spring force. Thus, for each gravitational wave produced by the cosmological body along the wavy path by certain velocity during compression there is identical one (same amplitude and frequency) produced by the same velocity in opposite direction during stretching. Accordingly, the gravitational waves produced by the oscillator during compression destruct those produced during stretching presenving self-sustaining of frictionless system. Gravitational waves produced by the oscillator just before or after passing the wave axis leave the oscillator system (energy loss) as they pushed by the other cosmological object that acts on the oscillator by the spring force in its direction along its ecliptic path. Afterwards, these gravitational waves re-enter the oscillator system (energy gain) by the oscillator once again preserving energy of the renewable oscillator system. Figure 6 shows the generated gravitational waves by the moon (silver arrows) and the earth (blue arrows) along their paths, and the energy loss from the wavy path of the moon by the earth (red arrows) that re-enter the wavy path again (energy gain) by the moon (green arrows) as detected from the sun (yellow).

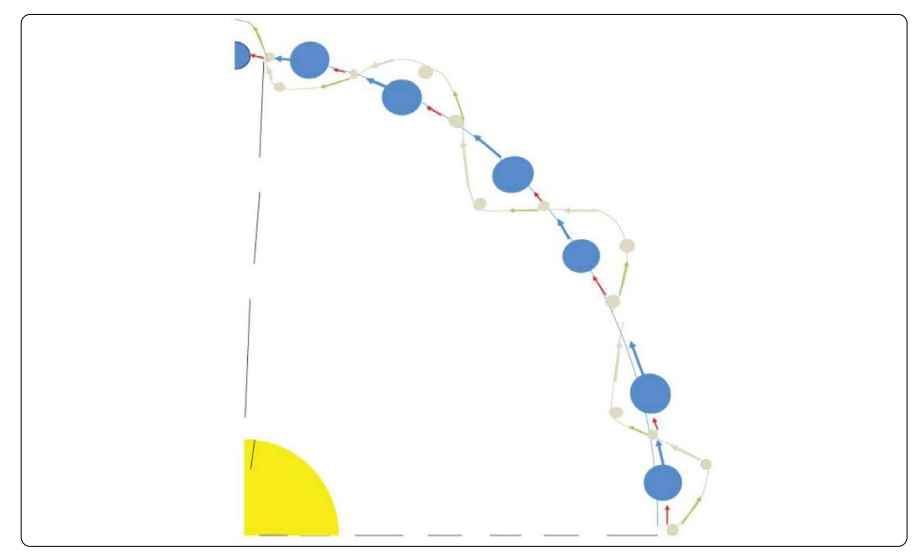

Figure 6 . shows the generated gravitational waves by the moon (silver arrows) and the earth (blue arrows) along their paths, and the energy loss from the wavy path of the moon by the earth (red arrows) that re-enter the wavy path again (energy gain) by the moon (green arrows) as detected from the sun (yellow). 
Accordingly, theory of the relative image predicts that gravitational waves observations of the cosmological bodies expresses those produced by the oscillator just before and after passing the wave axis (just before and after the spring force vanishes) only. Thus, observational gravitational waves generated by an oscillator whose simple harmonic motion expressed by $(x=$ Asin $\omega t)$ is predicted to be observed only when time (t) belongs to $\{0-, 0+, T-/ 2, T+/ 2, T-, T+, \ldots\}$ where $T$ is the periodic time of the simple harmonic motion. Gravitational-wave astronomy is an emerging branch of observational astronomy aims to use gravitational waves to collect observational data from the universe about the cosmological objects and their events. Although, various gravitational-wave observatories (detectors) such as advanced LIGO began observations in September 2015, gravitational waves are not easily detectable [7]. For instance, the detected power from the Earth-Sun system is about 200 watts which is truly tiny compared to the motion of the earth around the sun [8]. The perception of the gravitational waves provided by theory of the relative image is consistent with their tiny detected observations by LIGO; the velocity of the moon along its wavy path just before or after passing the elliptical path of the earth around the sun as detected from the sun in the perpendicular direction to the elliptical path is $1023 \mathrm{~m} / \mathrm{s}$. At these positions, the generated gravitational waves by the moon is pushed away (energy loss) the wavy path of the moon by the earth and re-enter (energy gain) the wavy path of the moon once again by the moon itself on its consequent crossing the elliptical path. Thus, if the power of the detected gravitational waves is 200 watt, then the force drives the moon at these positions is ( $F=$ Power/velocity) (200/1023) Newton.

Accordingly, the positions at which the gravitational force were detected are distant by $3.76 \times 10 * 13 \mathrm{~m}$ from the elliptical path of the earth around the sun, just before and after the intersection between paths of the moon and the earth as postulated by theory of the relative image [1].

\section{References}

1. Moawad EY. Theory of the Relative Image. J Space Explor. 2017; 6(2): 126.

2. Wieczorek $M$, Jolliff $B$, Amir K. The constitution and structure of the lunar interior. Rev Mineral Geoch. 2006; 60(1):221-364. doi: 10.2138/rmg.2006.60.3

3. Turnbull HW. Correspondence of Isaac Newton. Cambridge University Press. 1960.

4. Ehlers J. Survey of general relativity theory. in :Israel, Werner, relativity. Astrophysics and Cosmology. 1973; 1-125. doi: 10.1007/978-94-010-2639-0_1

5. Albert E, Nathan R. On gravitational waves. J Franklin Inst. 1937; 223(1): 43-54. doi: 10.1016/S0016-0032(37)90583-0

6. Serway RA, Jewett JWs. Physics for Scientists and Engineers. 7th ed. Tomson-Brooks/Cole Pacific Grove. 2008.

7. The Newest Search for Gravitational Waves has Begun. 2015.

8. Hawking SW, Israel W. General Relativity: An Einstein Centenary Survey. Cambridge University Press. 1979; 98. 